\title{
mid-infrared integrated photonics on a SiGe platform
}

\author{
L. Carletti, C. Blanchard, D.allioux, C. Monat, R. \\ Orobtchouk, P. Rojo-Romeo, Z. Lin, C. Jamois, J-L. \\ Leclercq, P. Viktorovitch, X. Letartre, C. Grillet \\ University of Lyon, Institut des Nanotechnologies de Lyon, \\ Ecole Centrale de Lyon \\ Ecully, France \\ Christian.grillet@ec-lyon.fr
}

\author{
M. Brun, S. Ortiz, P. Labeye, S. Nicoletti, \\ CEA-Leti MINATEC Campus, \\ GRENOBLE, France
}

\begin{abstract}
The mid-infrared is of great interest for a huge range of applications such as medical and environment sensors, security, defense and astronomy. I will give a broad overview of the different activities recently launched in INL Lyon, in close collaboration with several French and Australian institutions, under the umbrella of "Mid-IR integrated photonics" with a particular focus on novel integrated sources for the Mid-IR exploiting a nonlinear SiGe platform
\end{abstract}

Keywords-component; formatting; style; styling; insert (key words)

\section{INTRODUCTION (HEADING 1)}

The Mid-infrared (Mid-IR) wavelength range - from 2.5 to $20 \mu \mathrm{m}$ - is gaining at the moment a formidable momentum as it is experiencing a huge surge in interest for an enormous range of applications that affect almost every aspect of our society, from compact and highly sensitive biological and chemical sensors, homeland security applications, industrial monitoring (petro-chemical, down-well monitors, mining) imaging, geothermal imaging, defense, astronomy, to even sensing for the wine and grape industry, and many other fields. The reason is that the features contained in the Mid-IR absorption spectrum of all organic and many inorganic compounds relate directly to the vibrations of the bonds between atoms comprising the molecules of these compounds, which give rise to strong MidIR absorption spectra (molecular fingerprint) without the need for chemical modification. This fact has led to a drive to develop molecular sensing platforms using mid-IR light. One major roadblock to achieving such devices, however, is the lack of low-cost and easily deployable photonic technologies that operate in the mid-IR. Current mid-IR technologies used for monitoring and understanding our health or our environment are still based on bulky, mostly operating in free space, components and stand-alone single frequency laser sources that operate in relatively narrow spectral regions, thereby limiting the number of different molecules that can be detected with a single system. Those sensing platforms, to become ubiquitous, require compact and low-cost optical devices and, most importantly, optical sources that are widely

\author{
P. Ma, Y. Yu, B. Luther-Davies, D. Hudson, \\ S. Madden \\ CUDOS, Laser Physics Centre, Australian National \\ University, \\ Canberra, Australia
}

\author{
M. Sinobab, D. J. Moss \\ School of Electrical and Computer Engineering, RMIT \\ University, \\ Melbourne, Australia
}

tunable or yield a broadband emission so as to access the whole mid-IR band. To answer those requirements there have been calls over the past three years, for migrating the techniques developed in the near-IR especially within the Si photonics (and more generally the group IV photonics community) [1-4], to the mid-IR [5-11].

The mid-IR region has also been welcomed as a promising future regime for silicon based nonlinear photonic devices. The nonlinear loss phenomenon of two-photon absorption, that limits nonlinear optical applications in the near-infrared region, vanishes at these wavelengths [5,6], thus opening the door to photonic devices with new capabilities such as parametric waveguide amplifiers with positive net gain [10]. However, it has recently [12] been shown that, under some experimental conditions, e.g. using picosecond pulses, nonlinear losses in the mid-IR can still be significant. The silicon-on-insulator (SOI) material platform has attracted significant interest to implement on-chip integrated waveguides operating in the mid-IR thanks to the possibility of a CMOS compatible fabrication process. However, the increasing absorption of the silica cladding layer of the SOI at wavelengths longer than $3.5 \mu \mathrm{m}$ may limit the useful wavelength range of this material platform. It is therefore crucial to explore other material platforms. In this context, $\mathrm{SiGe}$ alloys on $\mathrm{Si}$ are seen as an attractive alternative platform to SOI for applications in the mid-IR due to their expected lower propagation losses and higher nonlinear response $[13,14]$.

Our aim at the Institut des Nanotechnologies de Lyon (INL) is to develop, building on the remarkable optical properties of group IV materials [[5][6], a chip-sized sensing platform by creating sources monolithically integrated on a CMOS compatible platform. To this end, we developed a comprehensive research program investigating at three different approaches to developing mid-IR integrated sources, including thermal (in collaboration with Charle-Fabry IOGS, CEA-LETI and delta-dore under the IDEE project), antimonide-based (in collaboration with IES-Montepllier, 3-5 lab and CEA-LETI under the MOREMIR project) and nonlinear Silicon-based sources. Here I focus on our recent 
advances, in collaboration with CEA-LETI, CUDOS ANU and RMIT, towards the creation of a CMOS compatible, highly tunable and broadband Mid-IR wavelength coherent light sources on-chip [15].

\section{SIGE WAVEGUIDES FABRICATION AND EXPERIMENTAL SETUP}

The $\mathrm{SiGe} / \mathrm{Si}$ step index ridge waveguides consist of a $\mathrm{Si}_{0.6} \mathrm{Ge}_{0.4}$ core surrounded by a $\mathrm{Si}$ cladding (Fig.1(a)). The devices were fabricated at CEA-Leti on a CMOS fabrication line.

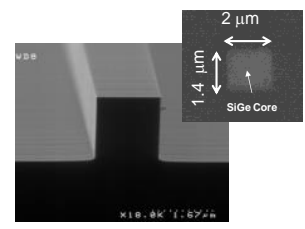

(a)

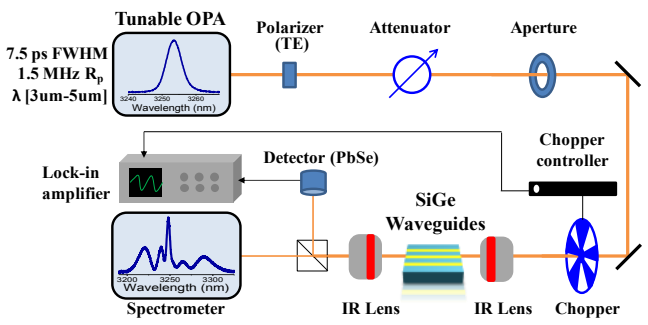

(b)

Fig. 1: (a) SEM image of a $\mathrm{SiGe}$ waveguide with an inset showing the $\mathrm{SiGe}$ core surrounded by Si cladding. (b) Experimental setup used for the transmission experiments.

The waveguides, with a core of dimension $1.4 \mathrm{~mm} \times 2 \mathrm{~mm}$ and length of $8.3 \mathrm{~cm}$, were obtained by first depositing a layer of $\mathrm{Si} 0.6 \mathrm{Ge} 0.4$ with a thickness of $1.4 \mathrm{~mm}$ on top of a $200 \mathrm{~mm}$ silicon wafer via reduced pressure CVD deposition. The experimental setup is depicted in Fig.1(b). Optical pulses in the picosecond regime $(7.5 \mathrm{ps}$ duration, repetition rate of $1.5 \mathrm{MHz})$ and in the femtosecond regime (320 fs duration, repetition rate of $21 \mathrm{MHz}$ ) were obtained from a TE-polarized and tunable optical parametric amplifier (OPA). The output signal power was measured by a $\mathrm{PbSe}$ detector while the output spectrum was measured with an optical spectrum analyzer. The insertion loss of the setup was $7 \mathrm{~dB}$, roughly constant over the experimental wavelength range.

\section{EXPERIMENTAL RESULTS AND DISCUSSION}

We report here only measurements performed in the picosecond regime. The measurements were systematically repeated at four different wavelengths in the mid-IR region: 3250nm, 3750nm, 4162nm, and 4750nm. The output power was measured with the PbSe detector while the spectrum at the output of the waveguide was measured with a scanning monochromator. Sample spectra are shown in Figs. 2(a)-2(d) for increasing powers. It can be seen that as the peak coupled intensity increased, more and more side lobes appeared, which is characteristic of SPM. The strong asymmetry in the spectral shape is attributed to free-carrier effects. A similar behavior is seen at all wavelengths used in these experiments. A rapid drop in the nonlinear transmission is observed as the input intensity increases, indicating the presence of nonlinear losses, and it is characteristic of multi-photon absorption.

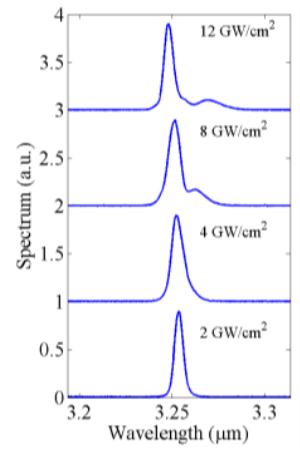

(a)

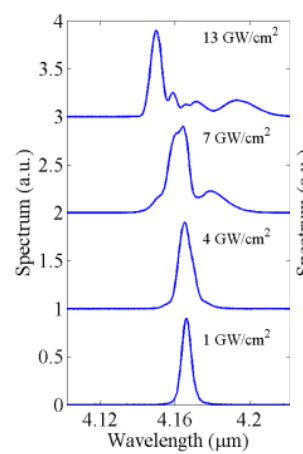

(c)

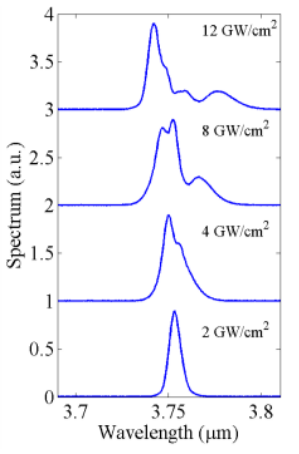

(b)

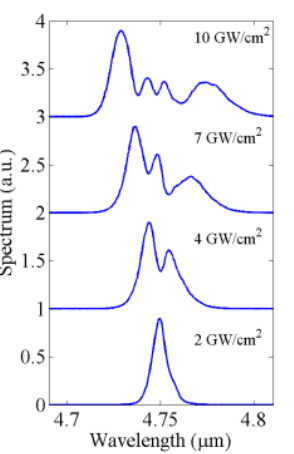

(d)
Fig. 2: (a-d) Normalized transmitted spectrum as a function of the coupled peak intensity for picosecond pulses centered at wavelengths between $3.25 \mu \mathrm{m}$ and $4.75 \mu \mathrm{m}$.

The three- and four-photon absorption coefficients of $\mathrm{Si} 0.6 \mathrm{Ge} 0.4$ were estimated and were found to be comparable to those of crystalline $\mathrm{Si}$ and $\mathrm{Ge}$. Furthermore, the nonlinear refractive index, $\mathrm{n} 2$, was estimated from spectral broadening measurements. We found that theory that accounts for freecarrier effects, multi-photon absorption and the Kerr effect was able to correctly predict the experimental results for peak intensities up to at least $5 \mathrm{GW} / \mathrm{cm} 2$, while by including higherorder nonlinear refractive effects arising from the real parts of $\chi(5)$ and $\chi(7)$ we obtained a better agreement with experiments for even higher pulse intensities.

\section{CONCLUSIONS}

We report measurements of the nonlinear optical response of low-loss $\mathrm{Si}_{0.6} \mathrm{Ge}_{0.4} / \mathrm{Si}$ waveguides in the mid-IR between $3250 \mathrm{~nm}$ and $4750 \mathrm{~nm}$. In this wavelength range, nonlinear 
losses due to multi-photon absorption and the associated generation of free-carriers are observed at high peak intensities. The three- and four-photon absorption coefficients of $\mathrm{Si}_{0.6} \mathrm{Ge}_{0.4}$ were estimated and were found to be comparable to those of crystalline $\mathrm{Si}$ and $\mathrm{Ge}$. Furthermore, the nonlinear refractive index, $\mathrm{n}_{2}$, was estimated from spectral broadening measurements. We found that theory that accounts for freecarrier effects, multi-photon absorption and the Kerr effect was able to correctly predict the experimental results for peak intensities up to at least $5 \mathrm{GW} / \mathrm{cm}^{2}$, while by including higherorder nonlinear refractive effects arising from the real parts of $\chi^{(5)}$ and $\chi^{(7)}$ we obtained a better agreement with experiments for higher pulse intensities. This suggests that the effect of these phenomena is not negligible in the wavelength range considered and at the pulse intensity levels used in the experiments. This could be caused by the fact that in the midIR we are close to the resonance frequencies of these higher order susceptibilities, which is also consistent with our observation of three- and four-photon absorption. These results represent the first experimental characterization of the nonlinear optical response of $\mathrm{Si}_{0.6} \mathrm{Ge}_{0.4} / \mathrm{Si}$ waveguides in the mid-IR and they will be useful for the design of future photonic devices based on this material platform and go a long way to understanding the optical nonlinear response of this material in the mid-IR wavelength range.

\section{ACKNOWLEDGMENT}

This research was supported by the People Program (Marie Curie Actions) of the European Union's Seventh Framework Program FP7/2007-2013 under REA grant agreements n ${ }^{\circ} \mathrm{PCI}-$ GA-2013-631543 and $\mathrm{n}^{\circ}$ PCIG10-GA-2011-304005, the French National Research Agency (ANR project MOREMIR) and was conducted within the context of the International Associated Laboratory "ALPhFA: Associated Laboratory for Photonics between France and Australia".

\section{REFERENCES}

[1] J. Leuthold, C. Koos, W. Freude, "Nonlinear silicon photonics," Nat. Photon. 4, 535-544 (2010).
[2] C. Monat, C. Grillet, M. Collins, A. Clark, J. Schroeder, C. Xiong, J. Li, L. O'Faolain, T. F. Krauss, B. J. Eggleton \& David J. Moss, " Integrated optical auto-correlator based on third-harmonic generation in a silicon photonic crystal waveguide", Nature Communications 5, 3246 (2014).

[3] B. Corcoran, C. Monat, C. Grillet, D. J. Moss, B. J. Eggleton, T. P. White, L. O'Faolain, and T. F. Krauss,"Green light emission in silicon via slow light enhanced third harmonic generation in photonic crystal waveguides," Nature Photonics 3, 206 (2009).

[4] C. Grillet, L. Carletti, C. Monat, P. Grosse, B. Ben Bakir, S. Menezo, J. M. Fedeli, D. J. Moss, "Amorphous silicon nanowires combining high nonlinearity, FOM and optical stability", Optics Express 20, 2260922615 (2012)

[5] R. A. Soref, "Mid-infrared photonics in silicon and germanium", Nature Photonics 4495 (2010).

[6] B. Jalali, "Silicon Photonics: Nonlinear optics in the mid-infrared," Nature Photonics 4, 506 (2010)

[7] X. Liu, R. M. Osgood Jr, Y. A. Vlasov, and W. M. J. Green, "Midinfrared optical parametric amplifier using silicon nanophotonic waveguides," Nat. Photonics 4, 557-560 (2010).

[8] G. Z. Mashanovich, M. M. Milošević, M. Nedeljkovic, N. Owens, B. Xiong, E. J. Teo, and Y. Hu, "Low loss silicon waveguides for the midinfrared," Opt. Express 19, 7112-7119 (2011).

[9] P. Y. Yang, S. Stankovic, J. Crnjanski, E. J. Teo, D. Thomson, A. a. Bettiol, M. B. H. Breese, W. Headley, C. Giusca, G. T. Reed, and G. Z. Mashanovich, "Silicon photonic waveguides for mid- and long-wave infrared region," J. Mater. Sci. Mater. Electron. 20, 159-163 (2009).

[10] B. Kuyken, X. Liu, R. M. Osgood, R. Baets, G. Roelkens, and W. M. J. Green, "Mid-infrared to telecom-band supercontinuum generation in highly nonlinear silicon-on-insulator wire waveguides.," Opt. Express 19, 20172-81 (2011)

[11] R. Shankar, I. Bulu, and M. Lončar, "Integrated high-quality factor silicon-on-sapphire ring resonators for the mid-infrared," Appl. Phys. Lett. 102, 051108 (2013)

[12] X. Gai, Y. Yu, B. Kuyken, P. Ma, S. J. Madden, J. Van Campenhout, P. Verheyen, and R. Baets, "Nonlinear absorption and refraction in crystalline silicon in the mid-infrared," Laser Photonics Rev. 1-11 (2013)

[13] N. K. Hon, R. Soref, and B. Jalali, "The third-order nonlinear optical coefficients of $\mathrm{Si}, \mathrm{Ge}$, and $\mathrm{Si} 1-\mathrm{xGex}$ in the midwave and longwave infrared," J. Appl. Phys. 110, 011301 (2011).

[14] K. Hammani, M. A. Ettabib, A. Bogris, A. Kapsalis, D. Syvridis, M. Brun, P. Labeye, S. Nicoletti, D. J. Richardson, and P. Petropoulos, "Optical properties of silicon germanium waveguides at telecommunication wavelengths," Opt. Express 21, 16690-16701 (2013)

[15] L. Carletti, P. Ma, Y. Yu, B. Luther-Davies, D. Hudson, C. Monat, R. Orobtchouk, S. Madden, D. J. Moss, M. Brun, S. Ortiz, P. Labeye, S. Nicoletti, and C. Grillet, "Nonlinear optical response of low loss silicon germanium waveguides in the mid-infrared," Opt. Express 23, 8261$8271(2015)$ 